\title{
Development of medium and small size photomultipliers for experiments in neutrino physics
}

\section{Sultim Lubsandorzhiev ${ }^{\mathbf{1}}$}

Institute for Nuclear Research of the Russian Academy of Sciences

Moscow, pr-t 60-letiya Oktyabrya 7a, Russia

E-mail: sultim@inbox.ru

\section{Andrey Sidorenkov}

Institute for Nuclear Research of the Russian Academy of Sciences Moscow, pr-t 60-letiya Oktyabrya 7a, Russia

E-mail: andreassx7@gmail.com

\section{Bayarto Lubsandorzhiev}

Institute for Nuclear Research of the Russian Academy of Sciences Moscow, pr-t 60-letiya Oktyabrya 7a, Russia

E-mail: lubsand@rambler.ru

\section{Sergey Belyanchenko}

Institute for Nuclear Research of the Russian Academy of Sciences Moscow, pr-t 60-letiya Oktyabrya 7a, Russia

E-mail:andreassx7@gmail.com

\section{Tatyana Izotova}

Institute for Nuclear Research of the Russian Academy of Sciences Moscow, pr-t 60-letiya Oktyabrya 7a, Russia

E-mail: andreassx7@gmail.com

\section{Valentin II'yevsky}

Institute for Nuclear Research of the Russian Academy of Sciences Moscow, pr-t 60-letiya Oktyabrya 7a, Russia

E-mail: andreassx7@gmail.com

\section{Nikita Ushakov}

Institute for Nuclear Research of the Russian Academy of Sciences Moscow, pr-t 60-letiya Oktyabrya 7a, Russia

E-mail: nikita.ushakoff@gmail.com

\section{Nima Lubsandorzhiev}

Scobeltsyn Institute of Nuclear Physics of Lomonosov Moscow State University Moscow, Leninskie gory 1, Russia

E-mail:nilubs@rambler.ru

\section{${ }^{1}$ Speaker}

(C) Copyright owned by the author(s) under the terms of the Creative Commons 


\title{
Dmitriy Voronin
}

Institute for Nuclear Research of the Russian Academy of Sciences Moscow, pr-t 60-letiya Oktyabrya 7a, Russia

E-mail: dmitriy.voronin@gmail.com

\section{Arslan Lukanov}

Institute for Nuclear Research of the Russian Academy of Sciences

Moscow, pr-t 60-letiya Oktyabrya 7a, Russia

E-mail: arslanlukanov@yandex.ru

\section{Dmitriy Lukanov}

Institute for Nuclear Research of the Russian Academy of Sciences Moscow, pr-t 60-letiya Oktyabrya 7a, Russia

E-mail: andreassx7@gmail.com

\begin{abstract}
A number of small-size photomultipliers have been developed by collaboration of INR RAS and MELZ-FEU for large-scale astroparticle physics experiments in particular for neutrino, gammaastronomy and cosmic ray physics experiments. The developed photomultipliers are of different sizes and optimized for use in Cherenkov and scintillation detectors. The photomultipliers are of different sizes and shapes. The largest photomultipliers have 3 inches photocathodes hemispherical and flat. We present also results of extensive studies of parameters of developed photomultipliers.
\end{abstract}

36th International Cosmic Ray Conference -ICRC2019-

July 24th - August 1st, 2019

Madison, WI, U.S.A. 


\section{Introduction}

Classical vacuum photomultipliers (PMTs) still continue to be work-horses of experimental physics. Indeed it is rather difficult to find physics experiments where PMTs are not used at all. The number of PMTs used in high energy and astroparticle physics experiments already amount to many dozens of thousands. In large scale astroparticle experiments there are presently two competing tendencies - to exploit large-size or middle/small-size PMTs. The former is more conventional approach and the latter is relatively recent one. For more details of such approaches see in [1]. The Hyper-Kamiokande project pursue more conventional approach planning to use more than 50k large area $(0.5 \mathrm{~m}$ in diameter) PMTs [2]. The middle/small-size PMTs approach has been pushed forward by Km3Net team [3]. They are going to use more than 150k PMTs of 3" size in their multi-PMTs optical modules. An intermediate approach was accepted by the JUNO experiment where $\sim 18 \mathrm{k}$ half a meter size MCP-PMTs and $\sim 25 \mathrm{k} 3$ " PMTs will be installed [4]. Simultaneous operation of large area and small PMTs will help to reach record energy resolution in the experiment and will be a first manifestation of "double calorimetry". Taking into account certain plans to use large number (more than $100 \mathrm{k}$ ) 3" PMTs in the PINGU project [5] at the South Pole one can see clearly tendencies of "revival" of interest for medium/small size PMTs in astroparticle physics experiments, in neutrino physics experiments in particular. Following such tendencies we started active development of a series of such PMTs with sizes of $76 \mathrm{~mm}, 30 \mathrm{~mm}$ and $20 \mathrm{~mm}$.

\section{3" (76 mm) PMTs}

A picture of 3" PMT is shown in Fig.1.

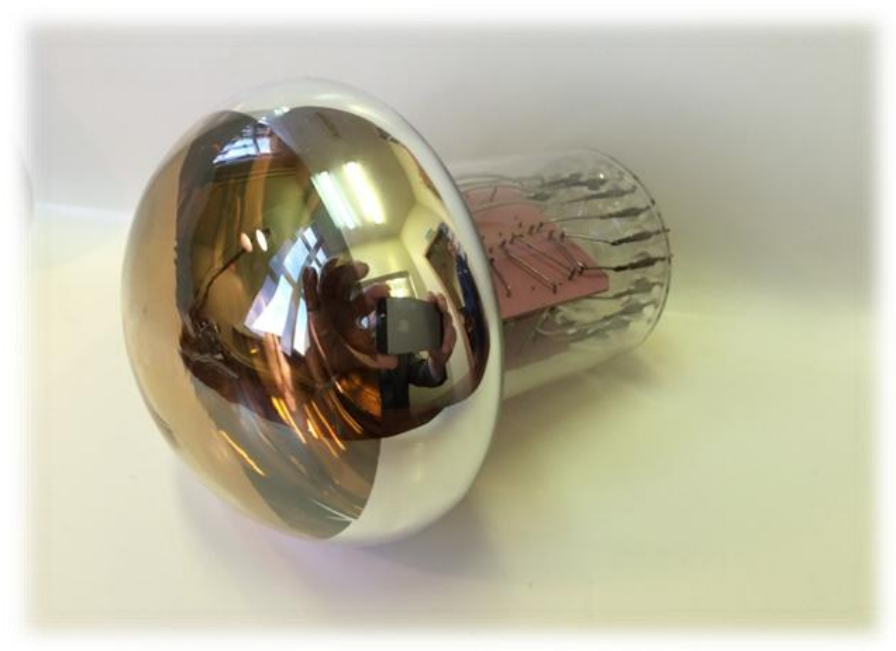

Fig.1. 3-inch PMT with mushroom shape glass envelope developed for large-sacle neutrino experiments. 
The PMT has a glass envelope of a mushroom shape and it is equipped with a bialkali photocathode with sensitivity in the range of $300-650 \mathrm{~nm}$. Its sensitivity maximum is reached at $\sim 380 \mathrm{~nm}$ where quantum efficiency is $\sim 33 \%$, see Fi.2.

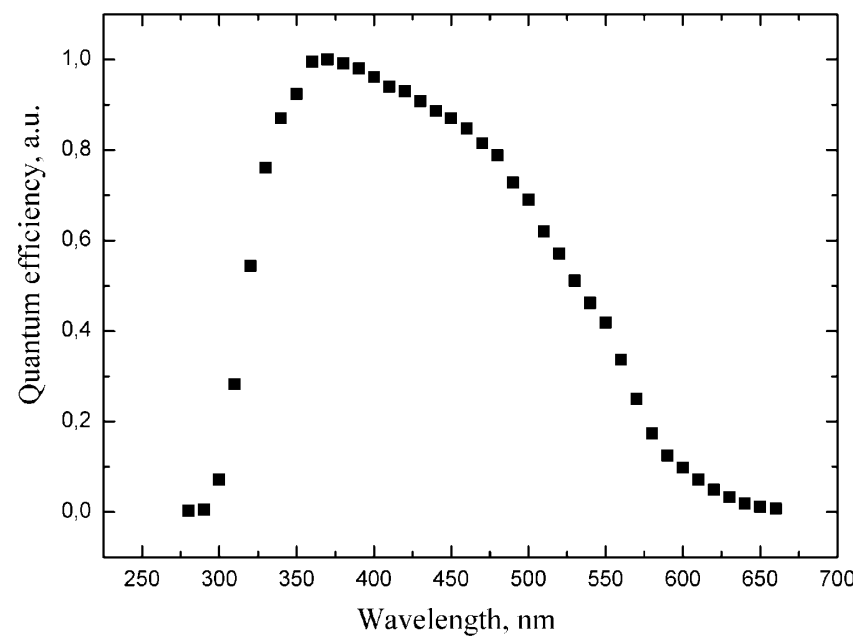

Fig.2. Dependence of photocathode quantum efficiency of 3" PMT on wavelength.

The photocathode sensitivity in the PMTs is characterized with good uniformity, see Fig.3, measurements are done into cross-sections oriented along and perpendicular to the surface of the first dynode of PMT. For the most part of the photocathode its non-uniformity is less substantially than $10 \%$.

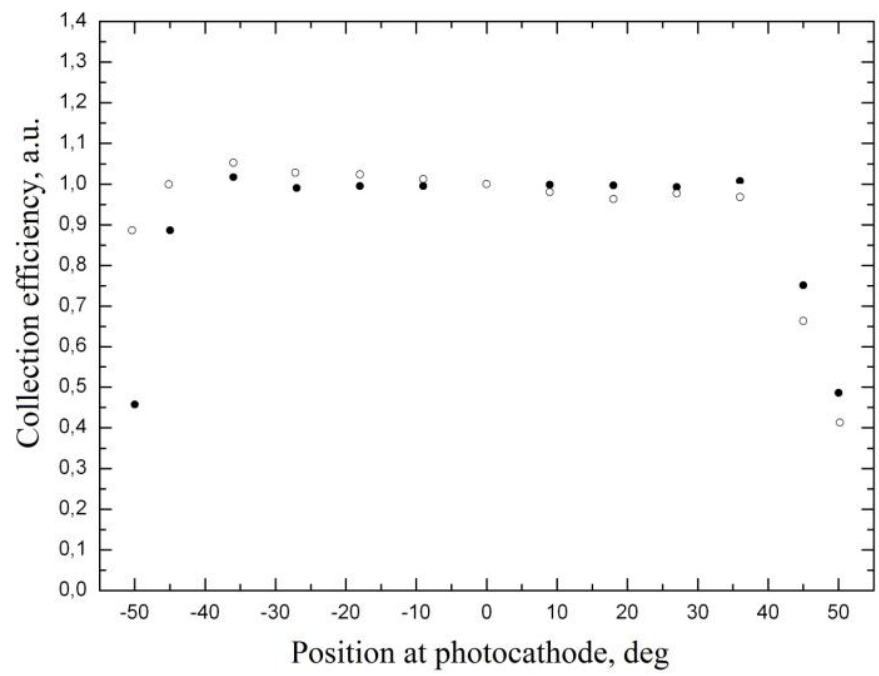

Fig.3. Uniformity of PMT photocathode. Full circles - measurements done along the first dynode surface, blank circles - perpendicular to it.

Exploiting in the PMT of large area high efficiency first dynode provides good single photoelectron response of the PMT, i.e. good single photoelectron resolution and small transit time spread (jitter) of single photoelectron pulses. Good single photoelectron response results in 
high level of effective quantum efficiency or photon detection efficiency of PMTs and high timing performance of PMTs.

In Fig.4 (left) typical charge spectrum of single photoelectron pulses of 3" PMT is shown. Single photoelectron resolution is $\sim 60-70 \%$ (FWHM). Charge spectrum of multi-photoelectron pulses is presented in Fig.4 (Right), contribution of four photoelectrons is clearly seen.
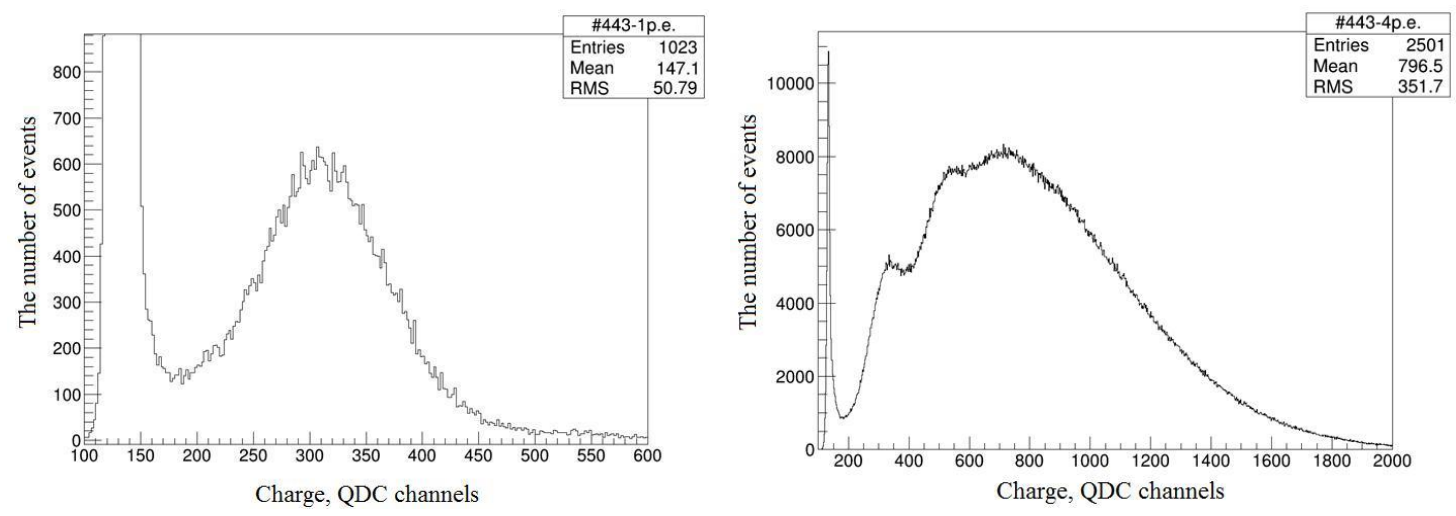

Fig. 4. Left - Typical charge distribution of single photoelectron pulses of 3" PMTs; Right - typical charge distribution of multi-photoelectron pulses of 3" PMTs.

Typical peak-to-valley ration of single photoelectron charge spectrum is more than 4. Typical dark current counting rate above threshold of $0.25 \mathrm{Q}_{1 \mathrm{pe}}$ is less than $1 \mathrm{kHz}$ at room temperature, $\mathrm{Q}_{1 \mathrm{pe}}$ - mean charge of single photoelectron pulses. Spectra shown in Fig.4 (Left) and 4 (Right) were measured with point-like illumination of the PMT photocathode at its pole.

The PMT's transit time spread was measured using fast LED driver with light pulses width $\sim 1 \mathrm{~ns}(\mathrm{FWHM})$ and $\lambda_{\max } \sim 405 \mathrm{~nm}$. The distribution of photoelectrons transit time under single photon illumination of the PMT's photocathode (jitter) is shown in Fig.5. There was a point-like illumination at the pole of the PMT photocathode.

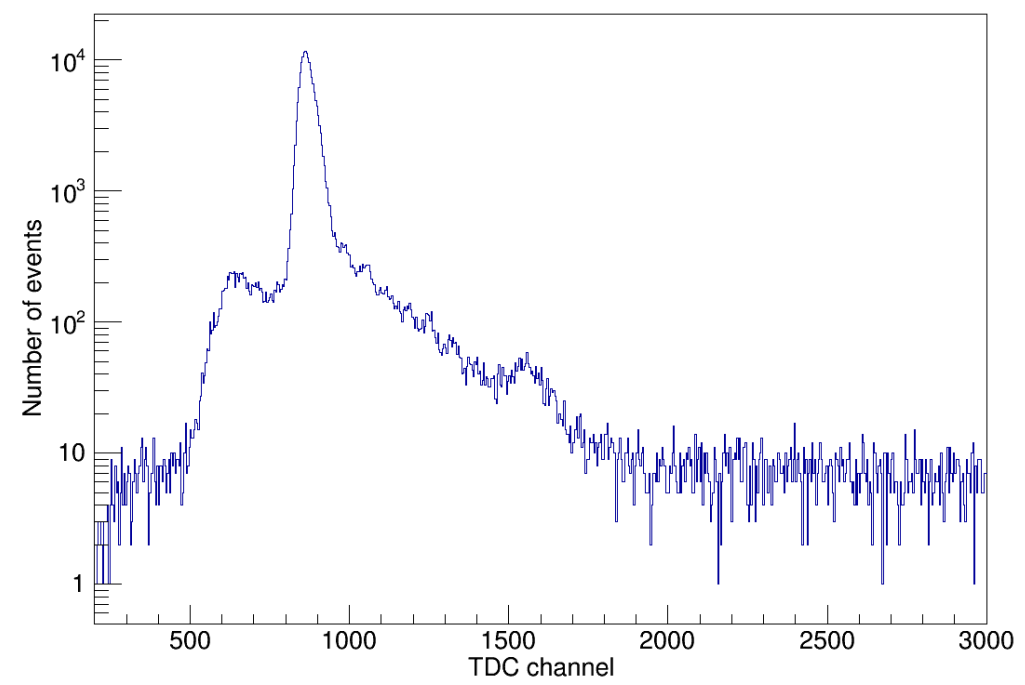

Fig.5. Photoelectron transit time distribution (jitter) for single photon illumination of the 3" PMT photocathode. 
The mean value of the photomultipliers TTS (jitter) is $3.7 \mathrm{~ns}$ (FWHM). The probabilities of prepulses and late pulses are less than $1 \%$ and $4 \%$ respectively.

The PMTs gain of $10^{7}$ is reached at operational voltage less than $1500 \mathrm{~V}$. Aftepulses probability at Gain of $10^{7}$ is less than $5-7 \%$ per one photoelectron by charge.

3" PMT with flat photocathode is under development too. The PMT is being developed for astroparticle physics experiments, including neutrino physics, cosmic ray physics and groundbased gamma astronomy. One possible application of the PMT is scintillator muon detectors. For such application high linearity of response is needed.

A picture of pilot samples of 3" PMTs demonstrated in Fig. 6.

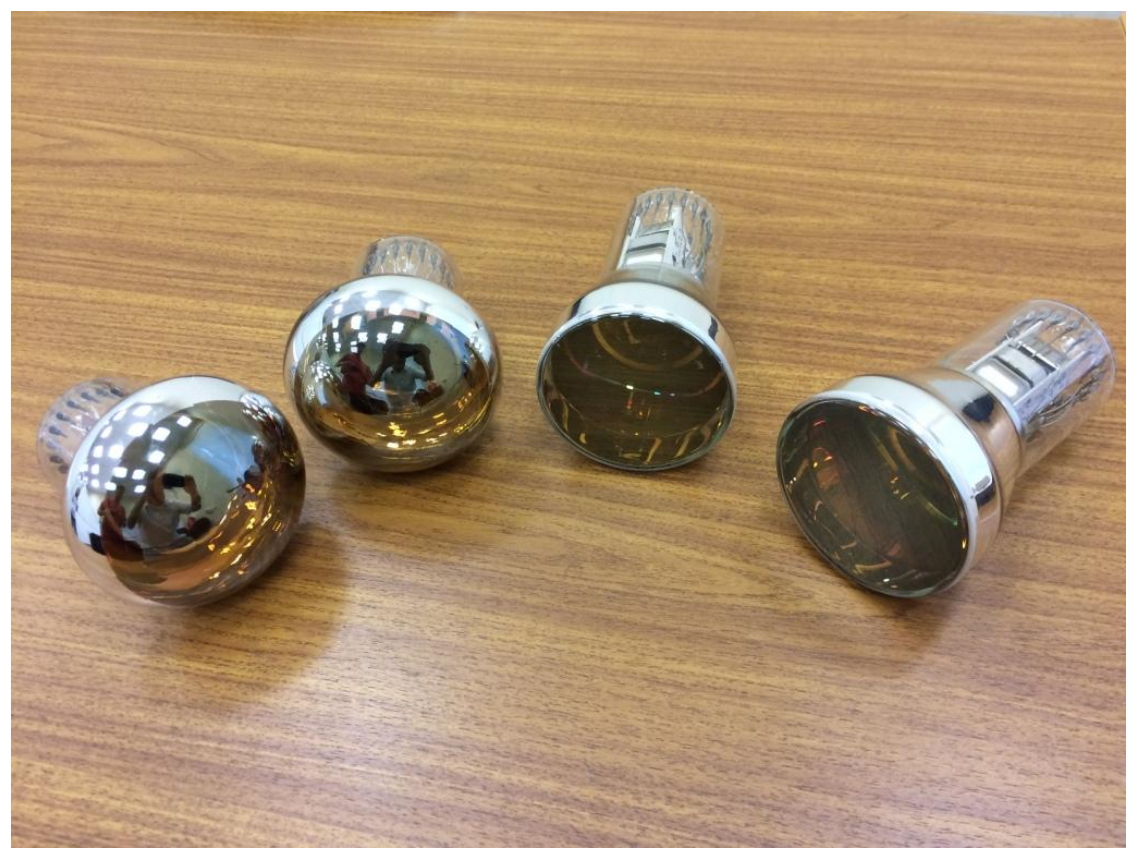

Fig. 6. Pilot samples of 3" PMTs under development.

\section{3. $20 \mathrm{~mm}$ and $30 \mathrm{~mm}$ PMTs}

Other issues of the development are $30 \mathrm{~mm}$ and $20 \mathrm{~mm}$ size PMTs. They are being developed for a number of astroparticle physics experiments - in neutrino physics, cosmic ray physics and ground based gamma astronomy among them.

They have also bialkali photocathodes with maximum sensitivity at $\lambda_{\max } \sim 380-400 \mathrm{~nm}$. Quantum efficiency at sensitivity maximum equals to $27-28 \%$.

Charge distributions of single and multi-photoelectron pulses measured for $20 \mathrm{~mm}$ PMT are shown in Fig. 7 (Left) and Fig. 7 (Right) correspondingly. Single electron resolution is $\sim 70 \%$ (FWHM). Peak-to-valley ratio of single photoelectron charge distribution is 3.5-4 and even more. In the multi-photoelectron spectrum even four photoelectrons peak is also clearly seen. 

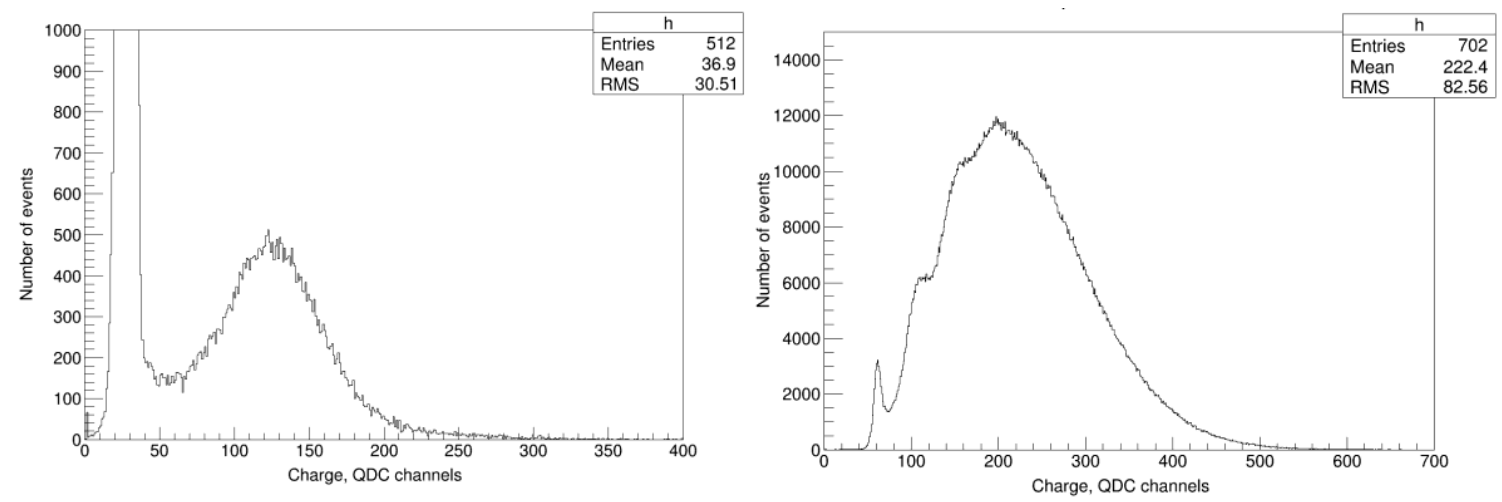

Fig. 7. Left - Charge distribution of single photoelectron pulses of $20 \mathrm{~mm}$ size PMT;

Right - Charge distribution of multi-photoelectron pulses of $20 \mathrm{~mm}$ size PMT.

Single photoelectrons transit time distribution has width of $1.8 \mathrm{~ns}$ (FWHM), Fig. 8. Probability of prepulses is less than $1 \%$. Due to small size of PMTs prepulses peak (around channel \#1650) practically merges with the main peak (around channel \#1700). Late pulses probability is less than $4 \%$. It is interesting that there is second late pulses peak near channel \#1900. Its origin will be clarified in further studies.

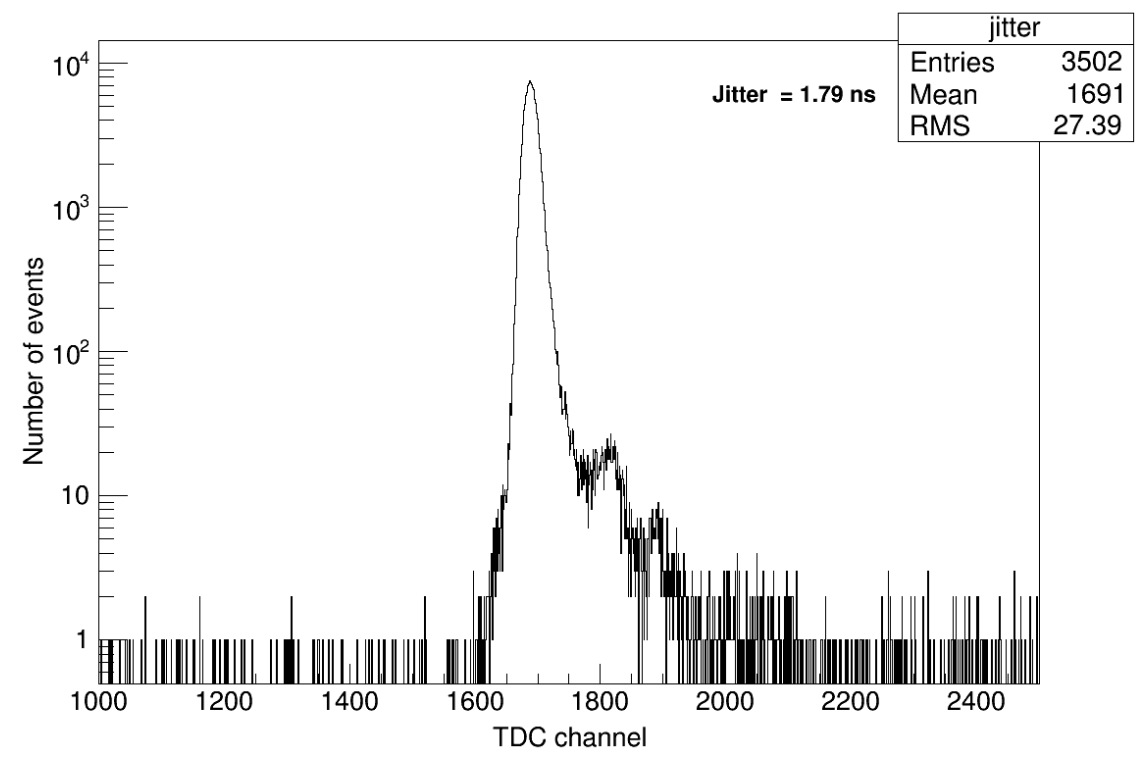

Fig. 8. Photoelectron transit time distribution (jitter) for single photon illumination of the $20 \mathrm{~mm}$ PMT photocathode.

10 -stages linear focused dynode system provides the gain of $(2-3) \times 10^{6}$ which is reached at operational voltage less than $1200 \mathrm{~V}$. The dark current counting rate is less than $1 \mathrm{kHz}$ at the gain of $(2-3) \times 10^{6}$ and at threshold of $0.25 \mathrm{Q}_{1 p e}$ and room temperature, here once again $\mathrm{Q}_{1 p e}$ is the mean charge of single photoelectron pulses at such gain. 
The afterpulses probability is less than $1-2 \%$ per one photoelectron. Time delay of afterpulses extends up to several microseconds.

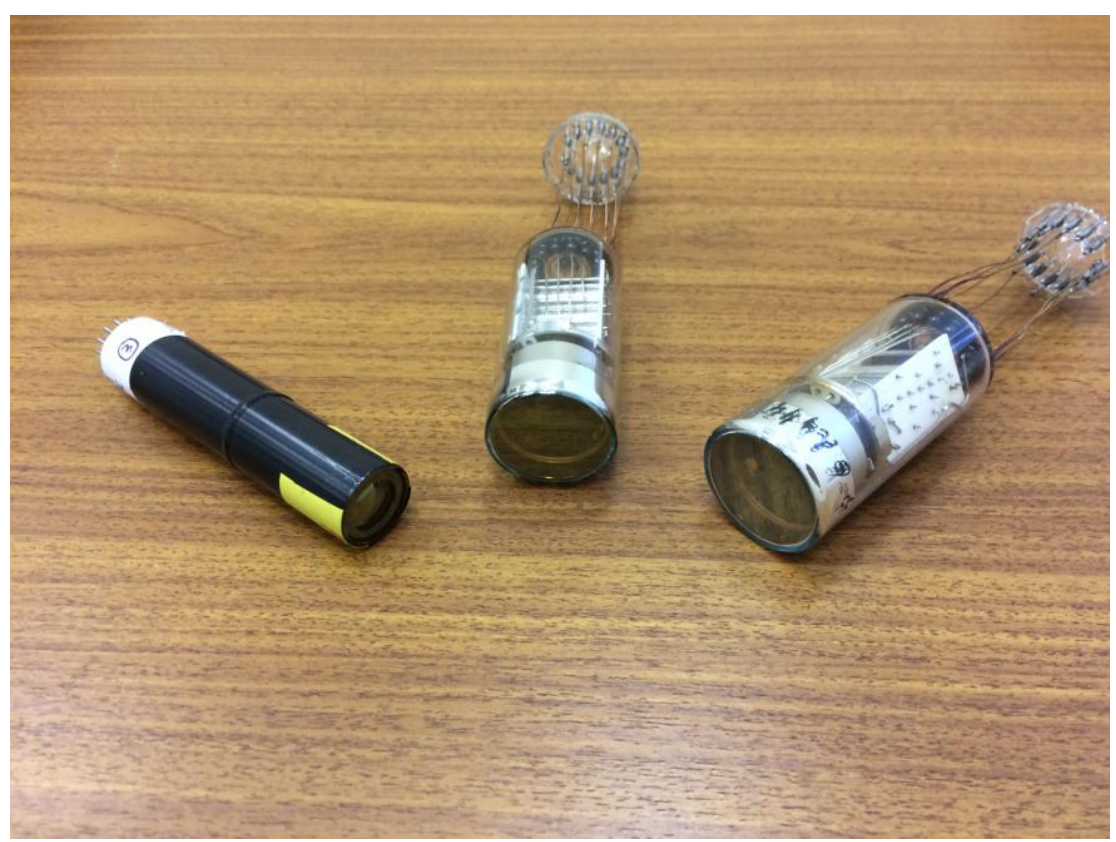

Fig. 9. Pilot samples of $20 \mathrm{~mm}$ and $30 \mathrm{~mm}$ PMTs under development.

\section{Conclusion.}

We are developing medium and small sizes photomultipliers for astroparticle physics experiments - neutrino physics, cosmic ray physics and ground-based gamma-astronomy among them. Studies of performances of the first pilot samples show good results and leave us optimistic for final results.

\section{Acknowledgements.}

The work was supported by the Russian Science Foundation grant no. 17-12-01331.

\section{References}

[1] B.K. Lubsandorzhiev. Evolution of ideas in photon detection // Physics of Particles and Nuclei. 2016. Vol.47. No.6. P.957-967.

[2] F. Di Lodovico et al. The Hyper-Kamiokande Experiment // Journal of Physics: Conference Proceedings. 2018. Vol.888. P.012020.

[3] P. Kooijman et al. (KM3NeT Collaboration). Multi-PMT Optical Module // Nuclear Instruments and Methods A. 2006. V.567. P.508.

[4] T. Adam et al. (JUNO Collaboration). JUNO conceptual design report // arxiv:1508.07166.

[5] D.J. Koskinen et al. Atmospheric neutrino results from IceCube-DeepCore and plans for PINGU // Journal of Physics: Conference Proceedings. 2018. Vol.888. P.012023. 